\title{
DC currents collected by a RF biased electrode quasi-parallel to the magnetic field
}

\author{
E. Faudot ${ }^{1, *}$, S. Devaux ${ }^{1}$, J. Moritz ${ }^{1}$, V. Bobkov ${ }^{2}$, and S. Heuraux ${ }^{1}$ \\ ${ }^{1}$ Institut Jean Lamour, Facult des sciences, 54500 Vandoeuvre-les-Nancy, France \\ ${ }^{2}$ Max-Planck-Institut fr Plasmaphysik, Boltzmannstrasse 2, D-85748 Garching, Germany
}

\begin{abstract}
Local plasma biasings due to RF sheaths close to ICRF antennas result mainly in a negative DC current collection on the antenna structure. In some specific cases, we may observe positive currents when the ion mobility (seen from the collecting surface) overcomes the electron one or/and when the collecting surface on the antenna side becomes larger than the other end of the flux tube connected to the wall. The typical configuration is when the antenna surface is almost parallel to the magnetic field lines and the other side perpendicular. To test the optimal case where the magnetic field is quasi-parallel to the electrode surface, one needs a linear magnetic configuration as our magnetized RF discharge experiment called Aline. The magnetic field angle is in our case lower than 1 relative to the RF biased surface. The DC current flowing through the discharge has been measured as a function of the magnetic field strength, neutral gas $(\mathrm{He})$ pressure and RF power. The main result is the reversal of the DC current depending on the magnetic field, collision frequency and RF power level.
\end{abstract}

\section{Introduction}

The negative DC currents measured on ICRF antenna structure [1] are driven by the self biasing of the plasma due to near RF electric fields and RF sheath. These DC currents can also be positive in some specific circumstances. The tilting angle (angle between magnetic lines and the wall or electrode surface) plays a role in the current collection as well as the RF biased surface compared to the connected wall surface on the other side of the flux tube. This study aims at understanding the main mechanisms responsible for the reversal of the current collected by a RF biased surface. To investigate the most favorable case to get positive current, we have biased

\footnotetext{
*Corresponding author:eric.faudot@univ-lorraine.fr
}

Table 1: Comparison of Tokamak and Aline parameters.

\begin{tabular}{l|l|l} 
& Tokamak (SOL) & Aline \\
\hline Density (m-3) & $10^{17}-10^{19}$ & $10^{14}-10^{17}$ \\
Ti $(\mathrm{eV})$ & $20-40$ & 0.026 \\
$\mathrm{Te}(\mathrm{eV})$ & $10-20$ & $1-10$ \\
$\mathrm{gas}$ & $\mathrm{H}, \mathrm{D}$ & $\mathrm{D}, \mathrm{He}, \mathrm{Ar}$ \\
$\mathrm{B}(\mathrm{T})$ & $1-5$ & $0-0.11$ \\
$\omega_{c i} / \omega_{p i}$ & 0.020 .8 & 00.4 \\
$\omega_{c e} / \omega_{p e}$ & 149 & 034 (for He) \\
$\omega_{r f} / \omega_{c i}$ & 2 & $10-100$ \\
$V_{r f} / T e$ & $1-100$ & $1-200$ \\
$\omega_{c i} / v_{i N}$ & $\gg 1$ & $0-10$
\end{tabular}

a RF cathode with a surface parallel to the magnetic field. This configuration can be found in our experimental setup Aline.

\section{Experimental setup : Aline}

Aline [2] is a linear magnetized plasma device dedicated to RF sheath study and plasma-wall interaction in RF magnetized discharges. The main advantages of this reactor is to be equipped with a broadband RF amplifier usually dedicated to RF communication antenna. This amplifier can sustain up to $400 \mathrm{~W}$ of reflected RF power, which allows to make it work without matching box to achieve a direct coupling as it is the case for RF biased structure close to ICRF antenna in Tokamak SOL. The second asset is the RF compensated probe (planar or cylindrical) mounted on a 3 axis movable arm, able to draw 3D map automatically in a volume $(50 \times 10 \times 10 \mathrm{~cm})$ surrounding the RF cathode. This allows to study the flux tubes connected to the cathode and the wall and investigate every topics linked to RF biased magnetized plasma. The typical plasma parameters in Aline can be compared to Tokamak edge plasma in table 1 . 


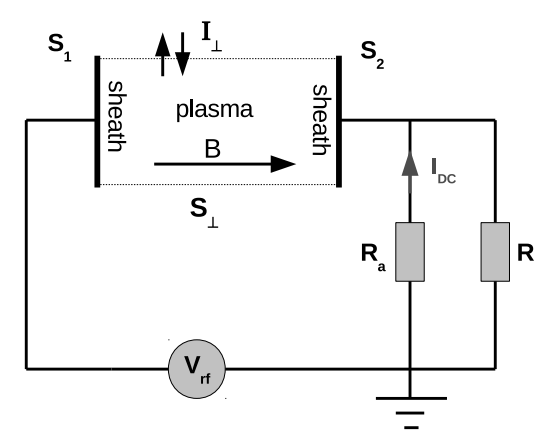

Figure 1: Electric schematic of the RF magnetized plasma discharge with direct coupling.

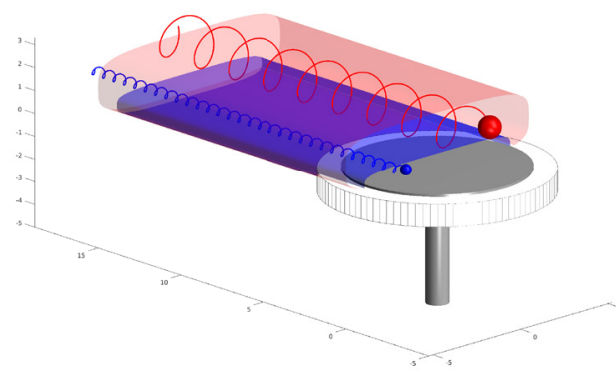

Figure 2: Ion (red) and electron (blue) magnetic flux tubes connected to the RF cathode.

\section{DC current measurement}

The DC current flowing in the electric circuit is measured according to schematic in figure 1. An ammeter is connected between the ground side of the amplifier and the wall chamber. Which means the ammeter measures a fraction of the total current flowing to the ground. To get the total current, one makes 2 measurements with 2 different ranges with their own internal resistance to deduce the resistance of the other branch. This measurement can not be done on the RF power side because the RF disturbs the measurement.

The DC current flows between the upper RF cathode surface $(\mathrm{R}=4 \mathrm{~cm})$ parallel to the magnetic field (tilting angle lower than 1) and the wall chamber. When the magnetic field is high enough the collecting surfaces are modified and can be sketched as in figure 2 .

3 parametric sweeps have been achieved.

The first is a magnetic field sweep as depicted in figure 3 for 3 values of the RF power $(-14 \mathrm{dBm}=27$ $\mathrm{W},-12 \mathrm{dBm}=43 \mathrm{~W},-10 \mathrm{dBm}=68 \mathrm{~W})$. One can see

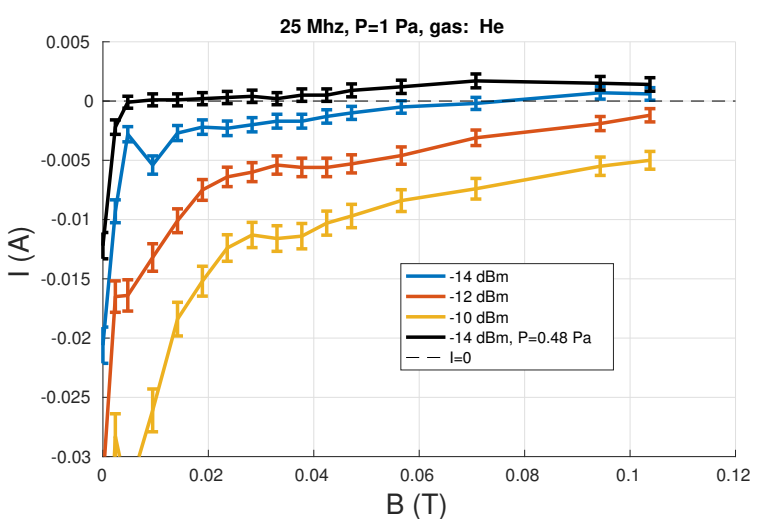

Figure 3: DC current with respect to $\mathrm{B}$ for 3 values of RF power and 2 pressures (both at $-14 \mathrm{dBm}$ ).

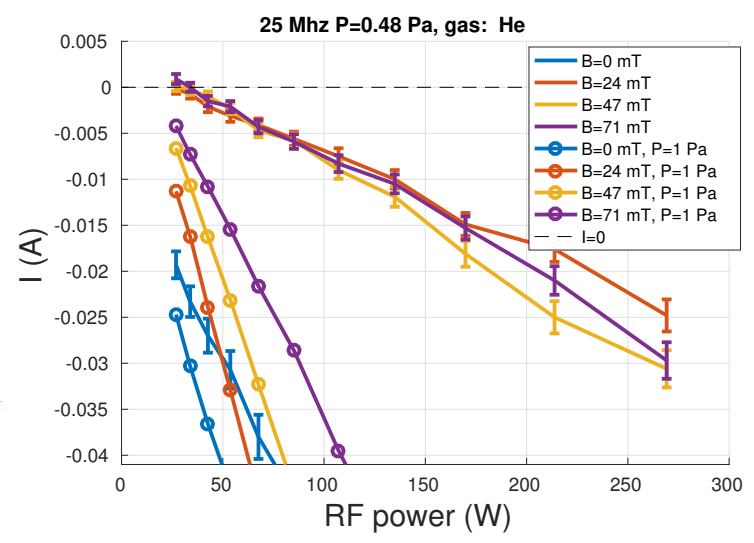

Figure 4: DC current with respect to RF power for 4 values of the magnetic field and 2 pressures.

that the current without magnetic field B is negative (electron current received by the cathode) but rises up very fast at low pressure to become positive for magnetic field on the order of $50 \mathrm{mT}$ at $0.48 \mathrm{~Pa}$ for $\mathrm{He}$ gas, and $100 \mathrm{mT}$ at $1 \mathrm{~Pa}$.

The second sweep (figure 4) is a RF power sweep for 4 values of the magnetic field. which shows that the higher the RF power and the higher is the negative current. The current is positive only for low RF power level (-14 dBm, or $27 \mathrm{~W}$ ), low pressure (only $0.48 \mathrm{~Pa}$ ) and high magnetic field (over $70 \mathrm{mT}$ ).

The third sweep (figure 5) is a pressure sweep for 4 values of the magnetic field. Again the current magnitude increases with the pressure and the current is negative except at low pressure (lower than 1 $\mathrm{Pa}$ ). At higher pressure, ions can be considered as unmagnetized because $\omega_{c i}<v_{i N}$. For example at 1 $\mathrm{Pa}$, the ion-neutral collision frequency is on the order of $200 \mathrm{kHz}(\mathrm{Ti}=2 \mathrm{eV})$, which corresponds to a magnetic field of $0.05 \mathrm{~T}$. But it is not easy to know the ion temperature at the edge of the flux tube where the RF electric field is strong. 


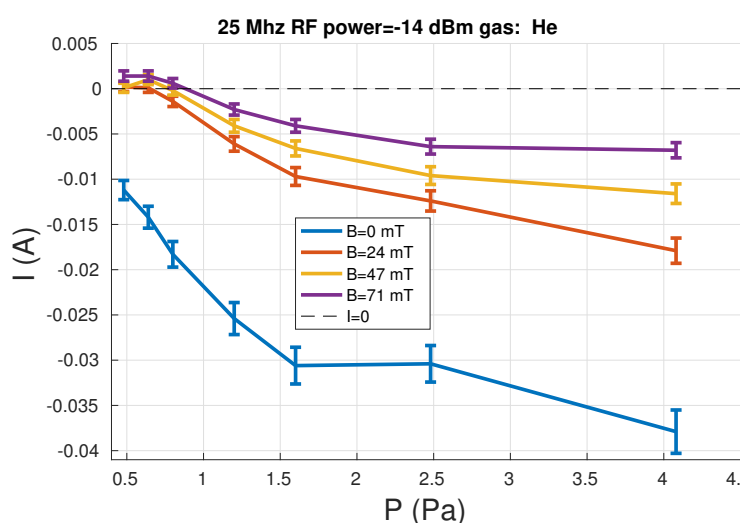

Figure 5: DC current with respect to pressure for 4 values of the magnetic field.

\section{Modeling}

This is a double asymmetric probe model with Boltzmann electrons. The model is based upon a previous model [3] assuming the surfaces of the cathode $S_{1}$ and anode $S_{2}$ are the same for both electrons and ions. While the classical symmetric double probe model can be written with a tanh function, the asymmetric model with perpendicular current depends on 4 collecting surfaces (figure 2 ) and one perpendicular current term whose nature is chosen as a function of the plasma type (discharge, fusion plasma [3]). The current conservation at both ends of the flux tube is mainly driven by classical RF sheath theory (unmagnetized) because the plasma potential is still higher than the average potential at the cathode $(0 \mathrm{~V})$, and then electrons are repelled by a negative potential well around the cathode. The saturation ion and electron currents are then deduced from the collecting surfaces. One can distinguish 4 collecting surfaces, with $D$ the cathode diameter, $r_{i}$ the ion Larmor radius and $r_{e}$ the electron Larmor radius :

$$
\begin{aligned}
& S_{1}^{i}=r_{i}\left(D+2 r_{i}\right) \quad S_{1}^{e}=r_{e}\left(D+2 r_{e}\right) \\
& S_{2}^{i}=S_{1}^{i} \quad S_{2}^{e}=S_{2}^{i}
\end{aligned}
$$

with $S_{1}^{i}, S_{1}^{e}, S_{2}^{i}$ and $S_{2}^{e}$ the collecting surface for ions and electrons at the cathode and at the wall respectively.

The DC current is then given by :

$$
I=j_{i} S_{1}^{i}\left(\frac{1-\frac{S_{1}^{e}}{S_{2}^{e}}\left(\frac{S_{2}^{i}}{S_{1}^{i}}+\frac{I_{\perp}}{j_{i} S_{1}^{i}}\right) \exp \left(\phi_{r f}(t)\right)}{1+\frac{S_{1}^{e}}{S_{2}^{e}} \exp \left(\phi_{r f}(t)\right)}\right)
$$

with $j_{i}$ the ion saturation current and $\phi_{r f}=$ e. $V_{r f} / T_{e} \cdot \sin (\omega t)$. This time averaged current over

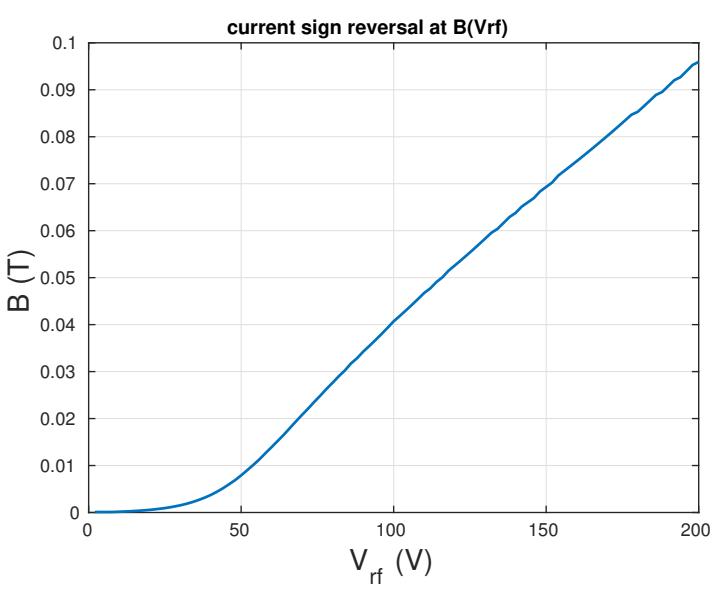

Figure 6: B field needed to reverse the current as a function of the RF potential according to equation 2 .

one RF period must be computed (integration not possible analytically). Assuming $S_{2}^{e}=S_{2}^{i}$ on the wall side because the surface is perpendicular to the magnetic field, this formula can be written as follows

$$
\begin{gathered}
I=j_{i} S_{1}^{i}\left(\frac{1-\exp \left(\phi_{r f}(t)+\delta \phi_{1}\right)}{1+\exp \left(\phi_{r f}(t)+\delta \phi_{2}\right)}\right) \\
\delta \phi_{1}=\ln \left(\frac{S_{1}^{e}}{S_{1}^{i}}+\frac{I_{\perp}}{j_{i} S_{1}^{i}}\right) \\
\delta \phi_{1}=\ln \left(\frac{S_{1}^{e}}{S_{2}^{e}}\right)
\end{gathered}
$$

From equation 2, we can deduce for which magnetic field the current is reversed at a given RF potential (collisionless model). Figure 6 reveals that at low RF potential only a small magnetic field is required while at higher RF potential the dependence is quasi linear. Above the curve the current sign is positive and below it is negative. From equation 3, one can see that the effect of the surface ratio and $I_{\perp}$ are similar to $\phi_{r f}$ effect.

The main effect of the perpendicular current is to shift the current (figure 7) so that it can sometimes never been reversed (at $-I_{\text {sat }}$ and $-2 I_{\text {sat }}$ ), with $I_{s a t}$ the ion satutation current at the cathode $\left(I_{s a t}=\right.$ $\left.e n_{0} C_{s} S_{1}^{i}\right)$. The sign of the perpendicular current is very important. In our device Aline, this current is supposed to be low and depends mainly on ionneutral collision [4]. This current sign is the same as the sign of the perpendicular electric field. If the perpendicular ion current enters into the flux tube, $I_{\perp}$ is negative as defined in assumptions and contributes to reverse the collected current at low $\mathrm{B}$, while a positive perpendicular current (higher than $I_{s a t}$ here) 


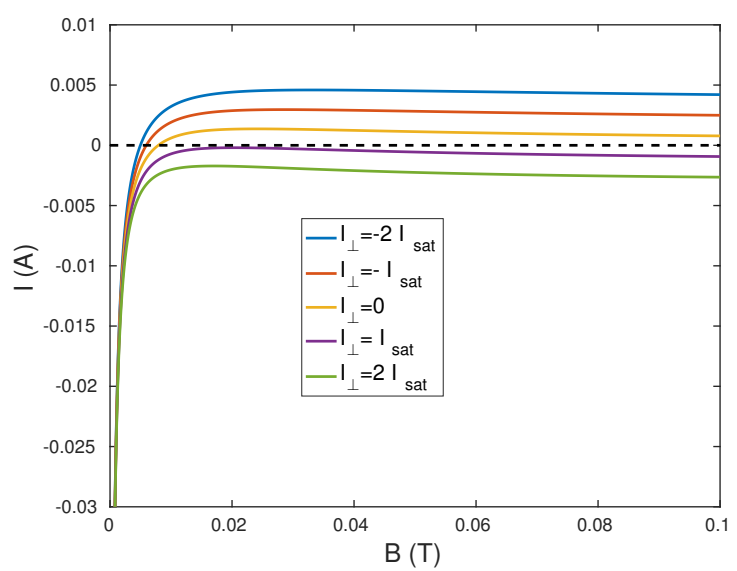

Figure 7: Effect of the perpendicular current on the I(B) characteristics.

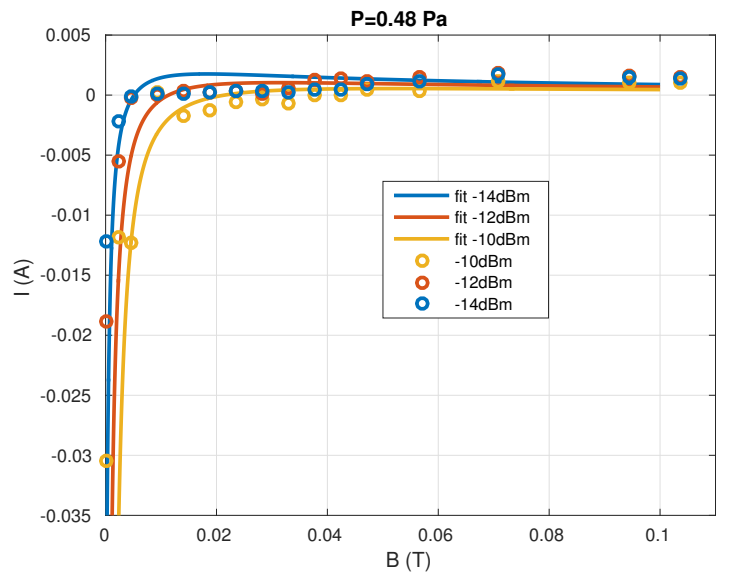

Figure 8: Fit of the $\mathrm{I}(\mathrm{B})$ characteristics according to equation 6 .

means that no positive current can be collected by the cathode.

Finally, in figure 8, the $\mathrm{I}(\mathrm{B})$ measured at $\mathrm{P}=0.48$ $\mathrm{Pa}$ and for 3 values of the rf power, can be fitted by equation 1 with the typical parameters measured by the probe and the rf potential measured by the coupler at the cathode. For the $-14 \mathrm{dBm}$ rf power, the fit does not match very well the measured data because the positive current first increases and next decreases unexpectedly according to measured values. The model still needs to be improved but gives the main physics involved in current reversal (RF potential, magnetic field). The pressure and thus the collisionnality should be added to the model to explain the pressure dependence.

\section{Conclusion}

The DC current collected on a RF biased surface is not necessarily negative as usually measured on the ICRF antenna structure in tokamaks or in RF capacitive discharges. When the magnetic field is high enough, the RF power at low level and at low pressure (low collisionnality), the DC current can be positive. This has been measured in the linear plasma device Aline in which the magnetic field, the gas pressure, the RF level and frequency can be swept as needed. These measurements have been compared to an asymmetric double probe model including perpendicular current and collecting surface for ions and electrons. The current reversal comes mainly from the collecting surface ratio on the RF and wall side for electrons and ions. If the surface ratio tends to one (symmetric case) and due to the higher mobility of ions perpendicular to the magnetic field, then the ion flux is higher on the RF biased structure. The optimal case is with a biased surface parallel to the magnetic field. The amount of positive current should again depend on the tilting angle, which could be investigated in a future work.

\section{References}

[1] R. Van Nieuwenhove and G. Van Oost, Plasma Phys. Controlled Fusion 34, 525 (1992)

[2] E. Faudot et al, Review of scientific instruments 86, 063502 (2015)

[3] E. Faudot, Physics of plasmas 22, 083506 (2015)

[4] V A Rozhansky et al, Nuclear Fusion, Vol. 39, No. 5 (1999) 613-628 